\title{
Prime elements in partially ordered groupoids applied to modules and Hopf algebra actions.*
}

\author{
Christian Lomp
}

January 8, 2004

\begin{abstract}
Primeness on modules can be defined by prime elements in a suitable partially ordered groupoid. Using a product on the lattice of submodules $\mathcal{L}(M)$ of a module $M$ defined in [3] we revise the concept of prime modules in this sense. Those modules $M$ for which $\mathcal{L}(M)$ has no nilpotent elements have been studied by Jirasko and they coincide with Zelmanowitz' "weakly compressible" modules. In particular we are interested in representing weakly compressible modules as a subdirect product of "prime" modules in a suitable sense. It turns out that any weakly compressible module is a subdirect product of prime modules (in the sense of Kaplansky). Moreover if $M$ is a self-projective module, then $M$ is weakly compressible if and only if it is a subdirect product of prime modules (in the sense of Bican et al.). An application to Hopf actions is given.
\end{abstract}

\section{Introduction}

Generalizing ring theoretic notions to modules often creates difficulties when they are of a multiplicative nature. If no obvious notion of a multiplication in a module is at hand, one often has to simulate the ring theoretic behaviour. However any such generalisation should coincide with the original one when applied to the ring itself.

In the following all rings $R$ will be associative with unit. We will refer to a unital left $R$-module simply as "module" if the context is clear. $\operatorname{End}_{R}(M)$ denotes the ring of $R$-endomorphisms of a module $M$ and we write endomorphisms on the opposite side to scalars. $\operatorname{Ann}_{R}(M)$ is the annihilator ideal of $M$ in $R$, i.e. the ideal consisting of all elements $x$ of $R$ such that $x m=0$ for all $m \in M$.

The concept of a prime ideal, resp. of a prime ring, is obviously a multiplicative notion. When we want to create a notion of primeness of a module $M$ it is natural to look first to the rings that are attached to $M$, i.e. $R / \operatorname{Ann}_{R}(M)$ and $\operatorname{End}_{R}(M)$. Let us briefly characterise when those rings are prime (resp. semiprime) in terms of the module $M$ :

Proposition 1.1 Let $R$ and $S$ be rings and $M$ be an $(R, S)$-bimodule. Assume that $M$ is a faithful left $R$-module. Then the following are equivalent:

(a) $R$ is a prime ring.

(b) for all submodules $N$ of $M: \operatorname{Ann}_{R}(N)=0$ or $\operatorname{Ann}_{R}(M / N)=0$.

*Work supported by Fundação para a Ciência e a Tecnologia through the Centro de Matemática da Universidade do Porto. Available as a PDF file from http://www.fc.up.pt/cmup. 
(c) for any $(R, S)$-subbimodule $N$ of $M$ that is $M$-generated as an $S$-module: $\operatorname{Ann}_{R}(N)=0$ or $\operatorname{Ann}_{R}(M / N)=0$.

Proof. $(a) \Rightarrow(b)$ Straightforward since $\operatorname{Ann}_{R}(N) \operatorname{Ann}_{R}(M / N) \subseteq \operatorname{Ann}_{R}(M)=0$. $(b) \Rightarrow(c)$ is trivial.

$(c) \Rightarrow(a)$ Let $I J=0$ for two ideals $I, J$ in $R$. Then $I \subseteq \operatorname{Ann}_{R}(J M)=0$ or $J \subseteq \operatorname{Ann}_{R}(M / J M)=0$ as $J M$ is a $(R, S)$-bimodule and $M$-generated as a right $S$-module.

In [19] J. E. Berg and R. Wisbauer called a module $M$ duprime if $M \in \sigma[N]$ or $M \in \sigma[M / N]$ for any submodule $N$ of $M$. Here $\sigma[X]$ is the full subcategory of $R$-Mod whose objects are submodules of factor modules of direct sums of copies of $X$. Since $\operatorname{Ann}_{R}(X) \subseteq \operatorname{Ann}_{R}(Y)$ holds whenever $Y \in \sigma[X]$ we get by Proposition 1.1(b) that any duprime module has a prime annihilator.

Interchanging $R$ and $S$ in Proposition 1.1 for $S=$ End $(M)$ we also can apply the proposition to determine when the endomorphism ring of a module is prime. The $(R$, End $(M))$-subbimodules are called fully invariant. Note that for a fully invariant submodule $N$ of $M$ we have $\operatorname{Ann}_{S}(N)=\operatorname{Hom}_{R}(M / N, M)$ and $\operatorname{Ann}_{S}(M / N)=\operatorname{Hom}_{R}(M, N)$. Since $\operatorname{Hom}_{R}(M, N) \neq 0$ holds for an $M$-generated $R$-submodule $N$, we get by $1.1(a) \Rightarrow(c)$ :

Corollary 1.2 The endomorphism ring of a left $R$-module $M$ is prime if and only if $\operatorname{Hom}_{R}(M / N, M)=0$ for all fully invariant $M$-generated submodules $N$ of $M$.

Several criteria for $\operatorname{End}_{R}(M)$ to be a domain are given by Bae Soon-Sook in [18]. Similar to Proposition 1.1 we can characterise when $R$ is semiprime:

Proposition 1.3 Let $R$ and $S$ be rings and $M$ be an $(R, S)$-bimodule. Assume that $M$ is a faithful left $R$-module. Then the following are equivalent:

(a) $R$ is a semiprime ring.

(b) for all submodules $N$ of $M: \operatorname{Ann}_{R}(N) \cap \operatorname{Ann}_{R}(M / N)=0$.

(c) for any $(R, S)$-subbimodule $N$ of $M$ that is $M$-generated as an $S$-module: $\operatorname{Ann}_{R}(N) \cap \operatorname{Ann}_{R}(M / N)=0$.

Proof. $(a) \Rightarrow(b)$ Straightforward since for any $N \subseteq M$ we have:

$\left(\operatorname{Ann}_{R}(N) \cap \operatorname{Ann}_{R}(M / N)\right)^{2} \subseteq \operatorname{Ann}_{R}(N) \operatorname{Ann}_{R}(M / N) \subseteq \operatorname{Ann}_{R}(M)=0$.

$(b) \Rightarrow(c)$ is trivial.

$(c) \Rightarrow(a)$ Let $I^{2}=0$ for an ideal in $R$. Then $I \subseteq \operatorname{Ann}_{R}(I M) \cap \operatorname{Ann}_{R}(M / I M)=0$ as $I M$ is an $(R, S)$-bimodule that is $M$-generated as a right $S$-module.

J. E. Berg and R. Wisbauer called a module $M$ dusemiprime if $M \in \sigma[N \oplus$ $(M / N)$ ] for every submodule $N$ of $M$. Since $\operatorname{Ann}_{R}(N \oplus(M / N))=\operatorname{Ann}_{R}(N) \cap$ $\operatorname{Ann}_{R}(M / N)$, we get by Proposition 1.1(b) that any dusemiprime module has a semiprime annihilator.

Interchanging left and right in Proposition 1.3 we also can apply the proposition to determine when the endomorphism ring of a module is semiprime.

Corollary 1.4 The endomorphism ring of a module $M$ is semiprime if and only if, for all fully invariant, $M$-generated submodules $N$ of $M$, if $f \in \operatorname{Hom}_{R}(M, N)$ and $N f=0$ then $f=0$. 


\section{Prime elements in partially ordered groupoids.}

The concept of a prime ideal of a ring just depends on the multiplication of (left) ideals in the ring and of the partial order of ideals. This allowed Birkhoff to carry the notion of prime ideals over to partially ordered sets that admit a multiplication, as follows. A partially ordered set $L$ is called a partially ordered groupoid (po-groupoid) if there exists a binary operation $\star: L \times L \rightarrow L$ such that for all $a, b, c \in L:$

$$
a \leq b \text { implies } a \star c \leq b \star c \text { and } c \star a \leq c \star b .
$$

If the operation $\star$ is associative, then $L$ is called a po-semigroup and if there exists an element $1 \in L$ with $a \star 1=a=1 \star a$ for all $a \in L$, then $L$ is called integral. An integral po-semigroup is simply called a po-monoid. If $L$ is a lattice then $L$ is called a $\ell_{0}$-groupoid and if moreover $\star$ distributes over join, i.e. for all $a, b, c \in L$ :

$$
a \star(b \vee c)=(a \star b) \vee(a \star c) \text { and }(b \vee c) \star a=(b \star a) \vee(b \star c),
$$

then $L$ is called a lattice ordered groupoid or $\ell$-groupoid for short.

A zero element of a po-groupoid $L$ is an element 0 which is the least element of $L$ with respect to $\leq$ and $a \star 0=0=0 \star a$ holds for all $a \in L$. An element $p \in L$ is called prime if $a \star b \leq p$ implies $a \leq p$ or $b \leq p$ for all $a, b \in L$ (see Birkhoff [4], [13] or [12]). An element $s \in L$ is called semiprime if $s$ is the lower bound of some prime elements $\left\{p_{\lambda}\right\}_{\Lambda}$ of $L$, i.e. $\forall \lambda \in \Lambda: s \leq p_{\lambda}$ and if for some $x \in L: x \leq p_{\lambda}$ for all $\lambda$ then $x \leq s$. In case $L$ is a lattice this means $s=\bigwedge p_{\lambda}$. The prime radical of $L$ is (if it exists) the lower bound of all prime elements of $L$. A po-groupoid $L$ with zero 0 is called prime if 0 is a prime element. A po-groupoid $L$ with zero 0 is called semiprime if 0 is a semiprime element.

In the sequel we want to compare the semiprime condition with the condition that there are no non-zero nilpotent elements. Since the notion of a nilpotent element involves the notion of a power of an element and since a power of an element in a not necessarily associative groupoid is not well-defined we give the following definition.

First we review the (recursive) definition of a binary tree. The empty tree $T=()$ is a binary tree and every expression $T=\left(T_{l}, T_{r}\right)$ is a binary tree where $T_{l}$ and $T_{r}$ are binary trees. $T_{l}$ (resp. $T_{r}$ ) will be called the left (resp. right) branch of $T$. The set of all finite binary trees is denoted by $\mathbb{T}$. The height of a tree $T$ is defined as 0 if $T=()$ and $\max (n, m)+1$ if $T=\left(T_{l}, T_{r}\right)$ where $n$ is the height of $T_{l}$ and $m$ is the height of $T_{r}$.

Definition 2.1 Let $L$ be a po-groupoid. For every $a \in L$ we define the map $\mu_{a}$ : $\mathbb{T} \rightarrow L$ by

$$
\mu_{a}(()):=a \text { and } \mu_{a}(T)=\mu_{a}\left(T_{l}\right) \star \mu_{a}\left(T_{r}\right) \text { for } T=\left(T_{l}, T_{r}\right) .
$$

Then any element in the image of $\mu_{a}$ is called a power of a.

If $L$ has a zero element 0 , then $a \in L$ is called nilpotent if 0 is a power of $a$. If the only nilpotent element of $L$ is 0 we say that $L$ is reduced.

We'll show that under suitable assumptions $L$ is reduced if and only if $L$ has no non-zero square-zero elements, i.e. no non-zero elements $a \in L$ such that $a^{2}:=$ $a \star a=0$.

The full binary tree $F_{n}$ of height $n$ is defined as follows: $F_{0}=()$ and $F_{n}=$ $\left(F_{n-1}, F_{n-1}\right)$ for $n \geq 1$. The following Lemma can be easily proved using induction.

Lemma 2.2 Let $L$ be a po-groupoid and $a \in L$ such that $a^{2} \leq a$. Then the following holds: 
1. $\mu_{a}\left(F_{n}\right) \geq \mu_{a}\left(F_{m}\right)$ for all $n \leq m$.

2. $\mu_{a}(T) \geq \mu_{a}\left(F_{n}\right)$ for all binary trees $T$ of height $n$.

Proof. (1) follows by induction and the hypothesis.

(2) is clear for $n=0$. Assume (2) has been proved for all $T$ of height $n$ for some $n \geq 0$. Let $T$ be of height $n+1$ and write $T=\left(T_{l}, T_{r}\right)$. Let $k$ be the height of $T_{l}$ and $m$ be the height of $T_{r}$. Then $n+1=\max (k, m)+1$ and so $n=\max (k, m)$. By induction and (1): $\mu_{a}\left(T_{l}\right) \geq \mu_{a}\left(F_{k}\right) \geq \mu_{a}\left(F_{n}\right)$ and $\mu_{a}\left(T_{r}\right) \geq \mu_{a}\left(F_{m}\right) \geq \mu_{a}\left(F_{n}\right)$. Hence

$$
\mu_{a}(T)=\mu_{a}\left(T_{l}\right) \star \mu_{a}\left(T_{r}\right) \geq \mu_{a}\left(F_{k}\right) \star \mu_{a}\left(F_{m}\right) \geq \mu_{a}\left(F_{n}\right)^{2}=\mu_{a}\left(F_{n+1}\right) .
$$

Corollary 2.3 Let $L$ be a po-groupoid with zero and let a be an element of $L$ such that $a^{2} \leq a$. If $a$ is a non-zero nilpotent element then there exists a non-zero power $b$ of a such that $b^{2}=0$.

Proof. Let 0 be a power of $a$. Then there exists a (non-empty) binary tree $T$ such that $\mu_{a}(T)=0$. Choose such a tree $T$ with minimal height, $k$ say. By the Lemma, $\mu_{a}(T) \geq \mu_{a}\left(F_{k}\right)$. Since $\mu_{a}(T)=0$ it follows that $\mu_{a}\left(F_{k-1}\right)^{2}=\mu_{a}\left(F_{k}\right)=0$. Taking $b:=\mu_{a}\left(F_{k-1}\right)$ gives the element desired. Since the height $k$ is minimal, $b \neq 0$.

As a corollary we get

Corollary 2.4 Let $L$ be a po-groupoid with zero 0 such that every element a satisfies $a^{2} \leq a$. Then $L$ is reduced if and only if $L$ has no non-zero square-zero elements.

In relation to semiprime po-groupoids we can now deduce

Corollary 2.5 A semiprime po-groupoid $L$ whose elements a satisfy $a^{2} \leq a$ is reduced.

Proof. By Corollary 2.4 it is enough to check that if $x \in L$ and $x^{2}=0$ then $x=0$. Since 0 is a lower bound for some set of prime elements $\left\{p_{\lambda}\right\}_{\Lambda}$ of $L$, we have $x^{2} \leq p_{\lambda}$ and so $x \leq p_{\lambda}$ for each $\lambda$. Hence $x=0$ as 0 is the lower bound of $\left\{p_{\lambda}\right\}_{\Lambda}$.

Under somewhat technical conditions we show that the converse is also true.

Recall that an element $c$ of a lattice $L$ is called compact if, whenever $c \leq \vee_{i \in I} a_{i}$, there exists a finite subset $F \subseteq I$ such that $c \leq \vee_{i \in F} a_{i}$. We say that an element $a \in L$ bounds an element $b \in L$ if $b \leq a$. An element $a$ of $L$ is called a left (resp. right) ideal if $b \star a \leq a$ (resp. $a \star b \leq a$ ) for all $b \in L$. Elements that are left and right ideals are called ideals.

Theorem 2.6 Let $L$ be an $\ell$-groupoid with zero 0 such that every element of $L$ is an ideal and bounds a non-zero compact element. Then $L$ is semiprime if and only if it is reduced.

Proof. If $L$ is semiprime, then it is reduced by Corollary 2.5. Now assume that $L$ is reduced. Set

$$
q:=\bigwedge\{p \in L \mid p \text { is a a prime element }\} .
$$

Assume $q \neq 0$. By hypothesis, $q$ bounds a non-zero compact element $0 \neq x_{1} \leq q$. Since $L$ is reduced, $x_{1}^{2} \neq 0$ and, since $x_{1}$ is an ideal, $x_{1}^{2} \leq x_{1}$. Again by hypothesis we may choose a non-zero compact element $0 \neq x_{2} \leq x_{1}^{2}$. Continuing this process 
we may obtain an infinite sequence of non-zero compact elements $\left\{x_{n}\right\}_{n \in \mathbb{N}}$ such that for all $n \in \mathbb{N}$ :

$$
x_{n+1} \leq x_{n}^{2} \leq x_{n}
$$

Now consider the set

$$
\Omega:=\left\{p \in L \mid \forall n \in \mathbb{N}: x_{n} \not \leq p\right\} .
$$

We shall apply Zorn's Lemma to obtain a maximal member of $\Omega$. First note that $\Omega$ is not empty, since $0 \in \Omega$. Let $\left\{p_{\lambda}\right\}_{\lambda \in \Lambda}$ be a chain in $\Omega$ and set $p:=\bigvee_{\lambda \in \Lambda} p_{\lambda}$. Assume that $x_{n} \leq p$ for some $n \in \mathbb{N}$. Then (since $x_{n}$ is compact) there is a $\lambda \in \Lambda$ with $x_{n} \leq p_{\lambda}$, a contradiction since $p_{\lambda} \in \Omega$. Hence $p \in \Omega$ and we can apply Zorn's Lemma to give a maximal member $p \in \Omega$.

Let us show that $p$ is actually a prime element of $L$. Assume $a \star b \leq p$ for some elements $a$ and $b$ of $L$. Then

$$
(a \vee p) \star(b \vee p)=(a \star b) \vee(p \star b) \vee(a \star p) \vee p^{2} \leq p
$$

since $L$ is an $\ell$-groupoid and $p$ is an ideal. Suppose that $a \vee p$ and $b \vee p$ are strictly above $p$, then (by the maximality of $p$ ) there exist $n, k$ such that $x_{n} \leq a \vee p$ and $x_{k} \leq b \vee p$. Without loss of generality we may assume $n \leq k$, then

$$
x_{k+1} \leq x_{k} \star x_{k} \leq x_{n} \star x_{k} \leq(a \vee p) \star(b \vee p) \leq p,
$$

impossible since $p \in \Omega$. Hence $a \vee p=p$ or $b \vee p=p$, i.e. $a \leq p$ or $b \leq p$. Hence $p$ is a prime ideal. But then $x_{n} \leq q \leq p$ for all $n$ - a contradiction to $p \in \Omega$. This shows that $q=0$. Hence $L$ is semiprime.

\section{The lattice of submodules of a module as a po- groupoid.}

Let $M$ be a left $R$-module and $S:=\operatorname{End}_{R}(M)$. We denote by $\mathcal{L}(M)$ the lattice of $R$-submodules of $M$ and by $\mathcal{L}(R)$ (resp. $\mathcal{L}(S)$ ) the lattice of left ideals of $R$ (resp. $S$ ). In order to define a prime notion on $M$ we are looking for a suitable "product" on the lattice of submodules $\mathcal{L}(M)$. One way to achieve this is by defining a product using maps from $\mathcal{L}(M)$ to $\mathcal{L}(R)$ (resp. $\mathcal{L}(S))$ and the multiplication in $R$ (resp. $S$ ) or the action of $R$ (resp. of $S$ ) on $M$.

For instance we define

$$
\begin{array}{lll}
\phi: & \mathcal{L}(M) \rightarrow \mathcal{L}(S) & \phi(N)=\operatorname{Hom}_{R}( \\
\psi: & \mathcal{L}(M) \times \mathcal{L}(S) \rightarrow \mathcal{L}(M) & \psi((N, I))=N I .
\end{array}
$$

Combining these maps and the action of $S$ on $M$ we get a product

$$
\mathcal{L}(M) \times \mathcal{L}(M) \stackrel{i d \times \phi}{\longrightarrow} \mathcal{L}(M) \times \mathcal{L}(S) \stackrel{\psi}{\longrightarrow} \mathcal{L}(M)
$$

This means concretely:

$$
N \star L:=\psi \circ(i d \times \phi)(N, L)=N_{H_{o m}}(M, L)=\sum\{(N) f \mid f: M \rightarrow L\} .
$$

This product has been defined in [3] and has the following properties:

Proposition 3.1 Let $M$ be an $R$-module and let $\star$ be as above.

1. $(\mathcal{L}(M), \star)$ is an $\ell_{0}$-groupoid with the submodule 0 as zero element.

2. All elements of $(\mathcal{L}(M), \star)$ are left ideals, i.e. $N \star L \subseteq L$ for all $N, L \in \mathcal{L}(M)$. 
3. For all submodules $N, K, L$ of $M$ the following hold:

(i) $M \star N=\sum\{\operatorname{Im}(f) \mid f: M \rightarrow N\}=$ : $\operatorname{Trace}(M, N)$;

(ii) $N \star M=N S$;

(iii) $N \star(L \star K) \subseteq(N \star L) \star K$;

(iv) $(N+L) \star K=(N \star K)+(L \star K)$;

(v) $N \star(K+L) \supseteq(N \star K)+(N \star L)$.

4. If $M$ is self-projective, then $(\mathcal{L}(M), \star)$ is an $\ell$-groupoid, i.e. $\star$ distributes over + .

5. If $M$ is projective in $\sigma[M]$, then $(\mathcal{L}(M), \star)$ is an $\ell$-semigroup, i.e. $\star$ is associative and distributes over + .

Proof. All conditions are easily verified. For (4) and (5) see [3].

We denote by $\mathcal{L}^{g}(M)$ the set of $M$-generated submodules of $M$ and by $\mathcal{L}_{2}(M)$ the set of fully invariant submodules of $M$, which are precisely the ideals in $\mathcal{L}(M)$. The set of $M$-generated submodules is invariant under right action of $\star$, i.e. if $N$ is $M$-generated, then $N \star L$ is $M$-generated for all $L$. The set of fully invariant submodules is invariant under left action of $\star$, i.e. if $N$ is fully invariant, then also $L \star N$ for all $L$. Moreover for any $N, L \in \mathcal{L}_{2}(M)$ we have $N \star L \subseteq N \cap L$. We let $\mathcal{L}_{2}^{g}(M):=\mathcal{L}^{g}(M) \cap \mathcal{L}_{2}(M)$ be the set of $M$-generated, fully invariant submodules of $M$. This is an integral $\ell_{0}$-groupoid with unit element $M$.

A module $M$ is called a multiplication module if every submodule $N$ of $M$ is of the form $I M$ for some two-sided ideal $I$ of $R$. Hence every submodule of $M$ is fully invariant, i.e. $\mathcal{L}(M)=\mathcal{L}_{2}(M)$. Moreover if $R$ is commutative, every submodule is $M$-generated since for any ideal $I$ and any $x \in I$ the map $\varphi_{x}: M \rightarrow I M$ with $\varphi_{x}(m):=x m$ is $R$-linear. Thus multiplication modules over commutative rings are self-generators whose submodules are fully invariant, i.e. $\mathcal{L}(M)=\mathcal{L}_{2}^{g}(M)$.

For any module $M$ and ideals $I$ and $J$ of $R$, the $\star$-product of $I M$ and $J M$ is contained in $I J M$ :

$$
(I M) \star(J M)=I M \operatorname{Hom}(M, J M)=I \operatorname{Trace}(M, J M) \subseteq I J M
$$

and the reverse inclusion is also easily established provided $M$ is a self-generator, i.e. Trace $(M, J M)=J M$. Hence we can describe the $\star$-product of submodules of multiplication modules:

Corollary 3.2 Let $M$ be a multiplication module which is a self-generator. Then

$$
N \star L=I J M
$$

for any submodules $N$ and $L$ of $M$ where $I$ and $J$ are ideals of $R$ such that $N=I M$ and $L=J M$.

We see that the product of submodules of multiplication modules over commutative rings as defined in [1] coincides with our $\star$-product. With our approach it is not necessary to show that this product is independent of the choice of representing ideals $I$ and $J$ for the submodules $N$ and $L$.

For any submodule $N \in \mathcal{L}(M)$, let $\operatorname{Rej}(M, N)$ be the reject of $N$ in $M$, i.e.

$$
\operatorname{Rej}(M, N):=\bigcap\{\operatorname{Ker}(f) \mid f \in \operatorname{Hom}(M, N)\} .
$$

Then it is easily verified that $\operatorname{Rej}(M, N)$ is the left annihilator of $N$ in the pogroupoid $(\mathcal{L}(M), \star)$, i.e. Rej $(M, N)$ is the largest submodule $T$ of $M$ with the property $T \star N=0$. Call $N \in \mathcal{L}(M)$ a right non-zero-divisor if $K \star N \neq 0$ for all $K \neq 0$. Thus we have $N$ is a right non-zero-divisor if and only if $\operatorname{Rej}(M, N)=0$ if and only if $M$ is $N$-cogenerated. 


\section{$4 \star$-Prime modules.}

Using the product $\star$ we now define prime elements and nilpotent elements in $\mathcal{L}(M)$.

A module $M$ is called retractable if $\operatorname{Hom}_{R}(M, N) \neq 0$ for all $0 \neq N \subseteq M$ (see [20]). Retractable modules can also be characterised by the property that Trace $(M, N)$ is essential in $N$ for any submodule $N$ of $M$.

Theorem 4.1 The following statements are equivalent for a left $R$-module $M$ with endomorphism ring $S$ :

(a) $(\mathcal{L}(M), \star)$ is a prime po-groupoid.

(b) every non-zero submodule of $M$ cogenerates $M$.

(c) for all non-zero submodules $N, L$ of $M: \operatorname{Hom}_{R}(M, N) \operatorname{Hom}_{R}(M, L) \neq 0$.

(d) $M$ is retractable and $f \operatorname{Hom}_{R}(M, M g) \neq 0$ for all $0 \neq f, g \in S$.

Proof. $(a) \Leftrightarrow(b) 0$ is prime if and only if every non-zero submodule is a right non-zero-divisor if and only if every non-zero submodule cogenerates $M$.

$(a) \Rightarrow(c)$ If $\operatorname{Hom}(M, N) \operatorname{Hom}(M, L)=0$ then $0=M \operatorname{Hom}(M, N) \operatorname{Hom}(M, L)=$ Trace $(M, N) \star L$ and hence $L=0$ or $\operatorname{Trace}(M, N)=M \star N=0$. Thus $L=0$ or $N=0$.

$(c) \Rightarrow(d)$ is clear.

$(d) \Rightarrow(a)$ Let $N$ and $L$ be two non-zero submodules of $M$. Since $M$ is retractable there are non-zero homomorphisms $f \in \operatorname{Hom}(M, N)$ and $g \in \operatorname{Hom}(M, L)$. By hypothesis $0 \neq M f \operatorname{Hom}(M, M g) \subseteq N \star L$.

Let us call a module that satisfies one of the above equivalent conditions a $\star-$ prime module. This is the definition of "prime" module used by Bican et al. in $[3]$.

Obviously by property $(d)$, every retractable module with prime endomorphism ring is $\star$-prime. When $M$ satisfies a projectivity condition $\star$-prime coincides with $M$ being a retractable module with prime endomorphism ring: A module $M$ is called semi-projective if any diagram

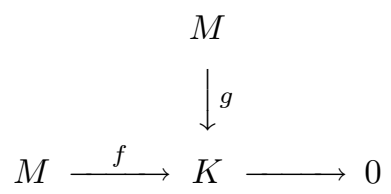

with $K \subseteq M$ can be extended by some endomorphism of $M$. In other words, $M$ is semi-projective if and only if for any endomorphism $f$ of $M$ we have $\operatorname{Hom}_{R}(M, M f)=$ $S f$ where $S=\operatorname{End}_{R}(M)$.

Proposition 4.2 A semi-projective module is $\star$-prime if and only if it is a retractable module with prime endomorphism ring.

It follows from an old result of Amitsur that torsionless modules over a prime ring are retractable and have a prime endomorphism ring. Recall that an $R$-module $M$ is torsionless if it is cogenerated by $R$.

Proposition 4.3 Every torsionless module over a prime (resp. semiprime) ring is retractable and has a prime (resp. semiprime) endomorphism ring. 
Proof. See [2, Theorem 27 and Corollary 21].

A module $M$ is called fully faithful if every non-zero submodule of $M$ is faithful. The "classical" notion of a prime module is the following: $M$ is prime if $M$ is a fully faithful $R / \operatorname{Ann}(M)$-module (see [11]). It is easy to see that $\star$-prime modules are prime and that the annihilator of a prime module is a prime ideal.

Using Amitsur's result we can show the following:

Corollary 4.4 The following statements are equivalent for a torsionless $R / \operatorname{Ann}(M)$ module $M$ :

(a) $M$ is retractable with prime endomorphism ring.

(b) $M$ is a $\star$-prime module.

(c) $M$ is a prime module.

(d) Ann $(M)$ is a prime ideal.

Proof. $(a) \Rightarrow(b) \Rightarrow(c) \Rightarrow(d)$ hold always.

$(d) \Rightarrow(a)$ By hypothesis $\bar{R}:=R / \operatorname{Ann}(M)$ is a prime ring and $M$ is a torsionless $\bar{R}$-module. By Amitsur's result Proposition $4.3 M$ is retractable and has a prime endomorphism ring.

The above corollary applies in particular to projective modules. Note that in general prime modules are not $\star$-prime modules as the $\mathbb{Z}$-module $\mathbb{Q}$ shows.

Recall that a ring $R$ is called left duo if every left ideal of $R$ is two-sided.

Theorem 4.5 The following statements are equivalent for a module $M$ over a left duo ring $R$ :

(a) $M$ is retractable with prime endomorphism ring.

(b) $M$ is ᄎ-prime.

(c) $M$ is cogenerated by $R / P$ for some $P \in \operatorname{Spec}(R)$.

Proof. $(a) \Rightarrow(b)$ holds by Theorem 4.1 .

$(b) \Rightarrow(c)$ holds by [16, Cor. 3.3.(1)].

$(c) \Rightarrow(a)$ holds by Proposition 4.3 .

We see that every $\star$-prime module over a commutative ring has a prime endomorphism ring. I have been unable to find an example of a $\star$-prime module whose endomorphism ring is not prime. Hence I state this as an

Open Problem: Find a $\star$-prime module whose endomorphism ring is not prime.

As with $\star$-prime modules, which were defined in terms of prime elements in the $\ell_{0}$-groupoid $(\mathcal{L}(M), \star)$, duprime modules were also initially defined using prime elments in a po-groupoid, as follows. Let $\mathbb{L}_{M}$ denote the set of all hereditary pretorsion sublasses $\alpha \subseteq \sigma[M]$ and let $\mathbb{L}_{M}^{o p}$ be $\mathbb{L}_{M}$ with reversed partial ordering. The product : of two classes $\alpha$ and $\beta$ is defined by

$$
\alpha: \beta:=\{X \in \sigma[M] \mid \exists A \subseteq X \text { with } A \in \alpha \text { and } X / A \in \beta\} .
$$

Berg and Wisbauer defined a module to be duprime if $\left(\mathbb{L}_{M}^{o p},:\right)$ is a prime $\ell$-groupoid.

This raises the question if the other prime notions, for instance the "classical" prime notion of Kaplansky, can be interpretted in the context of prime elements in po-groupoids.

Question: Does there exist a po-groupoid $L$ attached to a module $M$ such that $M$ is prime if and only if $L$ is a prime po-groupoid?

Prime multiplication modules are precisely those with prime annihilator: 
Proposition 4.6 Let $M$ be a multiplication module. Then $M$ is prime if and only if $\operatorname{Ann}_{R}(M)$ is prime.

Proof. The necessity is clear. Without loss of generality we might assume $\operatorname{Ann}_{R}(M)=$ 0 and $R$ being prime. For any submodule $N=I M$ where $I$ is an ideal of $R$, we have $\operatorname{Ann}_{R}(N)=\operatorname{Ann}_{R}(I)$. Since $R$ is prime and $\operatorname{Ann}_{R}(I) I=0$, we have $I=0$ or $\operatorname{Ann}_{R}(I)=\operatorname{Ann}_{R}(N)=0$. Hence $N=0$ or $\operatorname{Ann}_{R}(N)=\operatorname{Ann}_{R}(M)$, i.e. $M$ is prime.

Those multiplication modules which are $\star$-prime can be characterised as compressible modules. Recall that a module $M$ is called compressible if it can be embedded in each of its non-zero submodules.

Theorem 4.7 The following are equivalent for a multiplication module $M$ :

(a) $M$ is compressible;

(b) $M$ is «-prime;

(c) $M$ is retractable and satisfies one of the following statements:

(i) End $(M)$ is a domain;

(ii) $M$ is prime;

(iii) $\operatorname{Ann}_{R}(M)$ is a prime ideal.

Proof. The implications (a) $\Rightarrow(\mathrm{b}) \Rightarrow(\mathrm{c})(\mathrm{ii}) \Rightarrow(\mathrm{c})$ (iii) are always fulfilled and are easily verified.

We show $(\mathrm{c})($ iii) $\Rightarrow(\mathrm{c})(\mathrm{i})$. Let $f, g \in \operatorname{End}(M)$ with $g f=0$, i.e. $\operatorname{Im}(g) \subseteq \operatorname{Ker}(f)$. Choose ideals $I$ and $J$ such that $\operatorname{Im}(g)=I M$ and $(M) f=\operatorname{Im}(f)=J M$. Then

$$
0=(\operatorname{Im}(g)) f=I(M) f=I J M .
$$

Hence $I J \subseteq \operatorname{Ann}_{R}(M)$ and so, since $\operatorname{Ann}_{R}(M)$ is prime, either $0=I M=\operatorname{Im}(g)$ or $0=J M=\operatorname{Im}(f)$. Thus $f=0$ or $g=0$, i.e. End $(M)$ is a domain.

For (c)(i) $\Rightarrow$ (a) let $N$ be a non-zero submodule of $M$. Since $M$ is retractable, we may choose a non-zero $f \in \operatorname{Hom}_{R}(M, N)$. Since $g f=0$ for any $g \in \operatorname{Hom}_{R}(M, \operatorname{Ker}(f))$ and $\operatorname{End}(M)$ is a domain, we have $\operatorname{Hom}_{R}(M, \operatorname{Ker}(f))=0$. But as $M$ is retractable, $f$ must be injective and so $M$ can be embedded in $N$.

If $R$ is commutative, then every multiplication module is a self-generator and hence retractable. Thus the notions $\star$-prime, prime and compressible coincide for multiplication modules over commutative rings.

Multiplication modules over non-commutative rings appear in the study of algebras $A$ seen as bimodules over their multiplication algebra. Let $R$ be a commutative ring and let $A$ be an $R$-algebra with unit, but not necessarily associative. For any $a \in A$, let $L_{a}\left(\operatorname{resp} . R_{a}\right)$ denote the $R$-linear map $L_{a}(x)=a x\left(\operatorname{resp} . R_{a}(x)=x a\right)$ for $x \in A$. The multiplication algebra $M(A)$ of $A$ is the $R$-subalgebra of $\operatorname{End}_{R}(A)$ generated by the maps $L_{a}$ and $R_{a}$. A becomes a faithful unital cyclic left $M(A)$ module under the ordinary action of endomorphisms on $A$. Denote this action by , i.e. for $f \in M(A)$ and $a \in A$, we set $f \cdot a:=f(a)$. The left $M(A)$-submodules are precisely the two-sided ideals of $A$. Let $I$ be a two-sided ideal of $A$ and denote by $L(I)$ the ideal of $M(A)$ generated by the elements of the form $L_{x}$ where $x \in I$. One can show that $I=L(I) \cdot A$, i.e. $A$ is a multiplication module over the not necesarily commutative ring $M(A)$. Hence we get by Propositon 4.6 the known result that $A$ is a prime $M(A)$-module if and only if $M(A)$ is a prime ring. Note that the endomorphism ring $\operatorname{End}_{M(A)}(A)$ is naturally isomorphic to the centre $Z(A)$ of $A$ 
by the map $f \mapsto f(1)$. Thus $A$ is a retractable $M(A)$-module exactly when $A$ has a large centre, i.e. every non-zero ideal of $A$ contains a non-zero central element. By Theorem 4.7, $A$ is a $\star$-prime $M(A)$-module if and only if $A$ has a large centre and $M(A)$ is prime.

\section{Semiprime and weakly compressible modules.}

In this section we will discuss when $(\mathcal{L}(M), \star)$ is reduced, resp. semiprime.

Recall that a module $M$ is called weakly compressible if for any non-zero submodule $N$ of $M$ there exists an endomorphism $f: M \rightarrow N$ such that $f_{\mid N} \neq 0$ (see $[20])$.

Theorem 5.1 The following statements are equivalent for a left $R$-module $M$ with endomorphism ring $S$ :

(a) $(\mathcal{L}(M), \star)$ is a reduced po-groupoid.

(b) $\operatorname{Rej}(M, N) \cap N=0$ for all non-zero submodules $N \subseteq M$.

(c) $M$ is weakly compressible.

(d) $\operatorname{Hom}_{R}(M, N)^{2} \neq 0$ for all non-zero submodules $N \subseteq M$.

(e) $M$ is retractable and $f \operatorname{Hom}_{R}(M, M f) \neq 0$ for all $0 \neq f \in S$.

Proof. (a) $\Rightarrow$ (b) is clear since $(\operatorname{Rej}(M, N) \cap N)^{2}=0$.

(b) $\Rightarrow$ (c) Since $\operatorname{Rej}(M, N) \cap N=0, N \nsubseteq \operatorname{Rej}(M, N)$, i.e. there exists an $f: M \rightarrow N$ such that $N \nsubseteq \operatorname{Ker}(f)$.

(c) $\Rightarrow$ (d) By definition there exist homomorphisms $f: M \rightarrow N$ with $f_{\mid N} \neq 0$ and $g: M \rightarrow \operatorname{Im}(f)$ such that $g_{\mid \operatorname{Im}(f)} \neq 0$. Thus $0 \neq g f \in \operatorname{Hom}_{R}(M, N)^{2}$.

(d) $\Rightarrow$ (e) That $M$ is retractable is evident. If $0 \neq f \in S$, then $\operatorname{Hom}(M, M f)^{2} \neq$ 0 and so $f \operatorname{Hom}(M, M f) \neq 0$.

$(e) \Rightarrow(a)$ For all submodules $N$ of $M$ there exists a non-zero $f: M \rightarrow N$ such that $f \operatorname{Hom}_{R}(M, M f) \neq 0$. Hence $0 \neq M f \operatorname{Hom}_{R}(M, M f)=(M f) \star(M f) \subseteq N \star N$.

Hence we see that the semiprime notion with respect to our $\star$-product is precisely the notion of a "weakly compressible" module. This "semiprime" definition coincides with Jirasko's in [10], following [3]. As in the prime case we see that a retractable module with semiprime endomorphism ring is weakly compressible. In contrast to the $\star$-prime case, over commutative rings the converse is not true: if $M$ is the $\mathbb{Z}$-module $\mathbb{Z} \oplus \mathbb{Z}_{2}$, then $M$ is weakly compressible, but $\operatorname{End}_{\mathbb{Z}}(M)$ is not semiprime. More generally, the direct sum of weakly compressible modules is weakly compressible but, for modules $M$ and $N, \operatorname{End}_{R}(M \oplus N)$ is semiprime if and only if $\operatorname{End}_{R}(M)$ and $\operatorname{End}_{R}(N)$ are semiprime and $f \operatorname{Hom}_{R}(M, N) f \neq 0$ and $g \operatorname{Hom}_{R}(N, M) g \neq 0$ for all nonzero $f: N \rightarrow M$ and nonzero $g: M \rightarrow N$.

However, by Theorem 5.1, for a semi-projective module we get:

Corollary 5.2 A semi-projective module is weakly compressible if and only if it is a retractable module with semiprime endomorphism ring.

If $P$ is a prime ideal of a ring $R$, then $R / P$ is a prime module. It is well-known that a ring $R$ is semiprime if and only if the intersection of all its prime ideals is zero, i.e. $R$ is a subdirect product of the prime modules $R / P$. Hence one might consider modules that are subdirect products of prime modules as "semiprime", 
as was done by P. F. Smith and R. McClasland for the "classical" prime module concept.

In general subdirect products of prime modules don't have to be weakly compressible as the $\mathbb{Z}$-module $\mathbb{Q}$ shows. But the converse holds as we will see. First note the following Lemma:

Lemma 5.3 Let $M$ be a non-zero $R$-module and $P$ a fully invariant submodule of $M$. If $P$ is a prime element in $\left(\mathcal{L}_{2}(M), \star\right)$ then $M / P$ is a prime module.

Proof. Let $S$ be the endomorphism ring of $M$ and let $P \subseteq K \subseteq M$ for some submodule $K$ of $M$. Set $I=\operatorname{Ann}_{R}(K / P)$. Note that $I=\operatorname{Ann}_{R}(K S / P)$. Since $P$ is fully invariant and $(I M) \star(K S) \subseteq I K S \subseteq P$, we get

$$
(I M+P) \star(K S)=[I M \star(K S)]+[P \star(K S)] \subseteq P .
$$

Note that $I M+P$ and $K S$ are fully invariant submodule of $M$, i.e. elements of $\mathcal{L}_{2}(M)$. Since $P$ is a prime element in $\left(\mathcal{L}_{2}(M), \star\right)$ we get $I M \subseteq I M+P \subseteq P$ or $K \subseteq K S \subseteq P$, i.e. $I=\operatorname{Ann}_{R}(M / P)$ or $K=P$. Hence $M / P$ is a prime module.

Together with Theorem 5.1 and 2.6 we get as a corollary:

Corollary 5.4 Every weakly compressible module is a subdirect product of prime modules.

Proof. Let $M$ be weakly compressible. By Theorem $5.1(\mathcal{L}(M), \star)$ is a reduced po-groupoid. Hence also $\left(\mathcal{L}_{2}(M), \star\right)$ is reduced. All non-zero $N$ of $\mathcal{L}_{2}(M)$ are ideals and bound a compact element, e.g. if $0 \neq x \in N$ is any element, then $R x S \subseteq N$ is a compact element in $\mathcal{L}_{2}(M)$ where $S=\operatorname{End}(M)$. Hence by Theorem $2.6\left(\mathcal{L}_{2}(M), \star\right)$ is semiprime, i.e. there exist fully invariant submodules $P_{\lambda}$ for $\lambda \in \Lambda$ such that $0=\bigcap_{\lambda \in \Lambda} P_{\lambda}$. By the Lemma $M / P_{\lambda}$ is a prime module for all $\lambda$

Actually to conclude that $M$ is a subdirect product of prime modules we just need that $\left(\mathcal{L}_{2}(M), \star\right)$ is reduced. There are many "semiprime" notions for modules. We will sumarize them in the next Proposition. First of all recall some definitions. Jirasko called a module $M$ pseudo-semiprime if $N \cap \operatorname{Ann}_{R}(N) M=0$ for all $N \subseteq M$ (see [10]). Since $\operatorname{Ann}_{R}(N) M \subseteq \operatorname{Rej}(M, N)$ holds, we see that weakly compressible modules are pseudo-semiprime.

Proposition 5.5 Consider the following statements on a module $M$ :

(i) $M$ is retractable and has a semiprime endomorphism ring;

(ii) $M$ is weakly compressible;

(iii) every essential submodule of $M$ cogenerates $M$;

(iv) $\left(\mathcal{L}_{2}(M), \star\right)$ is semiprime.

(v) $M$ is a subdirect product of prime modules.

(vi) $M$ is pseudo-semiprime;

(vii) $\operatorname{Ann}_{R}(N)=\operatorname{Ann}_{R}(M)$ for every essential submodule $N$ of $M$;

(viii) $\operatorname{Ann}_{R}(M)$ is semiprime.

Then the implications $(i) \Rightarrow(i i) \Rightarrow($ iii $) \Rightarrow(i v) \Rightarrow(v) \Rightarrow(v i) \Rightarrow($ vii $) \Rightarrow$ (viii) hold. If $M$ is a torsionless as $R / \operatorname{Ann}(M)$-module then all statements $(i)-$ (viii) are equivalent. 
Proof. $(i) \Rightarrow(i i)$ by Theorem 5.1

(ii) $\Rightarrow$ (iii) By $5.1 N \cap \operatorname{Rej}(M, N)=0$ for any submodule $N$ of $M$. Hence every essential submodule $N$ cogenerates $M$.

(iii) $\Rightarrow($ iv) Let $N$ be a fully invariant submodule of $M$. Choose a complement $L$ of $N$, i.e. $L$ is maximal with respect to the property $L \cap N=0$. It is well-known that $N \oplus L$ is an essential submodule of $M$. Since $N$ is fully invariant, $N \star L \subseteq N \cap L=0$. Hence $N \subseteq \operatorname{Rej}(M, L)$. Assume $N \star N=0$, then $N \subseteq \operatorname{Rej}(M, N)$ and

$$
N \subseteq \operatorname{Rej}(M, N) \cap \operatorname{Rej}(M, L)=\operatorname{Rej}(M, N \oplus L)=0,
$$

since $N \oplus L$ cogenerates $M$ by hypothesis. Thus $\left(\mathcal{L}_{2}(M), \star\right)$ is reduced. By Theorem $2.6\left(\mathcal{L}_{2}(M), \star\right)$ is semiprime.

$(i v) \Rightarrow(v)$ follows from the proof of 5.4.

$(v) \Rightarrow(v i)$ Let $\left\{P_{\lambda}\right\}_{\Lambda}$ we submodules such that $M / P_{\lambda}$ is prime and $\bigcap_{\Lambda} P_{\lambda}=0$. Let $N$ be a submodule of $M$. Set $\Lambda^{\prime}:=\left\{\lambda \in \Lambda \mid N \nsubseteq P_{\lambda}\right\}$. For all $\lambda \in \Lambda^{\prime}$ we have $\operatorname{Ann}\left(\left(N+P_{\lambda}\right) / P_{\lambda}\right)=\operatorname{Ann}\left(M / P_{\lambda}\right)$. Hence

$\operatorname{Ann}_{R}(N) M \subseteq\left[\bigcap_{\lambda \in \Lambda^{\prime}} \operatorname{Ann}_{R}\left(\left(N+P_{\lambda}\right) / P_{\lambda}\right)\right] M \subseteq \bigcap_{\lambda \in \Lambda^{\prime}} \operatorname{Ann}_{R}\left(M / P_{\lambda}\right) M \subseteq \bigcap_{\lambda \in \Lambda^{\prime}} P_{\lambda}$.

Thus $N \cap \operatorname{Ann}_{R}(N) M \subseteq\left(\bigcap_{\Lambda \backslash \Lambda^{\prime}} P_{\lambda}\right) \cap\left(\bigcap_{\Lambda^{\prime}} P_{\lambda}\right)=0$.

$(v i) \Rightarrow(v i i)$ is clear.

(vii) $\Rightarrow$ (viii) Without loss of generality we may assume that $M$ is faithful. Let $I$ be an ideal of $R$ such that $I^{2}=0$. Choose a submodule $N$ of $M$ maximal with respect to the property that $N \cap(I M)=0$. Then $N \oplus(I M)$ is an essential submodule of $M$. By hypothesis $0=\operatorname{Ann}_{R}(N \oplus(I M))$. Since $I N \subseteq N \cap(I M)=0$, we get $I \subseteq \operatorname{Ann}_{R}(N \oplus(I M))=0$.

(viii) $\Rightarrow(i)$ if $M$ is a torsionless $R / \operatorname{Ann}(M)$-module, then by Amitsur's result Proposition $4.3 \operatorname{End}_{M}()$ is semiprime and $M$ is retractable.

For multiplication modules over a commutative ring we can show that the conditions above are all equivalent:

Proposition 5.6 Let $M$ be a multiplication module. Then $M$ is weakly compressible if and only if $M$ is retractable and $\operatorname{Ann}_{R}(M)$ is a semiprime ideal.

Proof. Let $f \in \operatorname{End}_{R}(M)$ and choose an ideal $I$ of $R$ such that $\operatorname{Im}(f)=I M$. If $f^{2}=0$ then

$$
0=(\operatorname{Im}(f)) f=(I M) f=I(M) f=I^{2} M .
$$

Hence $I^{2} \subseteq \operatorname{Ann}_{R}(M)$. As $\operatorname{Ann}_{R}(M)$ is semiprime, $I \subseteq \operatorname{Ann}_{R}(M)$, i.e. $f=0$, i.e. $\operatorname{End}_{R}(M)$ is reduced. By Theorem $5.1 M$ is weakly compressible.

Whether every weakly compressible module is a subdirect product of $\star$-prime modules is not known to me. However in case the module is self-projective we may apply 2.6 .

Proposition 5.7 The following statements are equivalent for a self-projective module $M$ :

(a) $M$ is a subdirect product of $\star$-prime modules;

(b) $M$ is weakly compressible;

(c) $\left(\mathcal{L}_{2}^{g}(M), \star\right)$ is a reduced integral $\ell$-groupoid. 
Proof. $(a) \Rightarrow(b)$ Assume $M$ is a subdirect product of $\star$-prime modules. Let $\left\{P_{\lambda}\right\}_{\Lambda}$ be a family of submodules such that each $M / P_{\lambda}$ is $\star$-prime. Since $M$ is self-projective, we have by $[3,2.7]$ that the $P_{\lambda}$ are prime elements in $\mathcal{L}(M)$. Hence $\mathcal{L}(M)$ is a semiprime $\ell$-groupoid and reduced by 2.5 , i.e. $M$ is weakly compressible. (b) $\Rightarrow(c)$ by Proposition $5.5\left(\mathcal{L}_{2}(M), \star\right)$ is semiprime. Hence also $\left(\mathcal{L}_{2}^{g}(M), \star\right)$ is reduced.

$(c) \Rightarrow(a)$ The hypotheses of 2.6 are fulfilled. Hence 0 is the intersection of prime elements $P_{\lambda} \in \mathcal{L}_{2}^{g}(M)$. Since each $P_{\lambda}$ is fully invariant and $M$ is self-projective, by $[3,2.6]$ each $M / P_{\lambda}$ is a $\star$-prime module, i.e. $M$ is a subdirect product of $\star$-prime modules.

Note that if $(N \star N) \star M=N \star(N \star M)$ holds for all $N \subseteq M$ then $(d) \Rightarrow(b)$ since in this case

$$
(N \star M) \star(N \star M)=N \star(N \star M)=(N \star N) \star M,
$$

i.e. $N^{2}=0$ if and only if $(N \star M)^{2}=0$. But as $N \star M$ is fully invariant, we get $M$ is weakly compressible, i.e. $\mathcal{L}(M)$ is reduced, if and only if $\mathcal{L}_{2}(M)$ is reduced.

Open Problem: 1.) Find a weakly compressible module which is not a subdirect product of $\star$-prime modules.

2.) Find a module that is cogenerated by each of its essential submodules, but which is not weakly compressible.

\section{Prime and Semiprime abelian groups}

In this section we want to determine the abelian groups that have the previously considered prime properties.

Faithful prime abelian groups coincide with the torsionfree abelian groups. The faithful $\star$-prime abelian groups $M$ are precisely the torsionless abelian groups, i.e. those embeddable in a direct product of infinite cyclic groups. As noted, this is equivalent to $M$ being retractable with prime endomorphism ring.

The nonfaithful prime abelian groups are precisely those that are isomorphic to a direct sum of copies of $\mathbb{Z}_{p}$. In the nonfaithful case, prime and $\star$-prime abelian groups coincide.

The following theorem of Samsonova [17] characterises the weakly compressible abelian groups:

Proposition 6.1 The following statements are equivalent for an abelian group $M$ :

(a) $M$ is a subdirect product of $\star$-prime

(b) $M$ is weakly compressible

(c) $T(M)$ elementary abelian and $M / T(M)$ is torsionless. modules.

Note that $M=\mathbb{Q}$ is prime, and hence a subdirect product of prime modules, but not weakly compressible.

We now characterise the abelian groups that are subdirect products of prime abelian groups. Dauns called a module $M$ semiprime if $a$ Ram $=0$ implies $a m=0$ for all $m \in M$ and $a \in R$. In other words the annihilator of each element $m$ of $M$ is a semiprime left ideal in the sense of Koh. In case $R$ is commutative, $M$ is semiprime in the sense of Dauns if and only if the annihilator of each submodule of $M$ is a semiprime ideal (see [7] or [6]). Note that any pseudo-semiprime module is semiprime in the sense of Dauns. For abelian groups the concepts of Dauns and Jirasko coincide: 
Proposition 6.2 The following statements are equivalent for an abelian group $M$ :

(a) $M$ is a subdirect product of prime abelian groups.

(b) $M$ is pseudo-semiprime.

(c) $M$ is semiprime in the sense of Dauns.

(d) $T(M)$ is elementary abelian.

In [9] Jenkins and Smith give an example of a module over a commutative ring that is semiprime in the sense of Dauns, but which is not a subdirect product of prime modules.

Bearing in mind that the "classical" notion of prime says that every non-zero submodule has the same annihilator as the module itself, a natural "semi" version of this notion is the restriction of this condition to essential submodules. In this case a module $M$ is defined to be semiprime if every essential submodule of $M$ has the same annihilator as $M$ (see Lemma 5.5(d)). This notion seems to be very weak, since it is easily seen that over an integral domain any module that is not torsion must be semiprime in this sense. Moreover this class is closed under direct products and direct sums, but may not be closed under direct summands. For instance if $M=\mathbb{Z} \oplus(\mathbb{Z} / 4 \mathbb{Z})$ then $M$ is semiprime in this sense, since any essential submodule of $M$ would intersect $\mathbb{Z} \oplus 0$ and hence contains a torsionfree element making its annihilator zero. On the other hand $\mathbb{Z} / 4 \mathbb{Z}$ is not semiprime.

For abelian groups we can characterise this property as follows:

Proposition 6.3 An abelian group $M$ has the property that every essential subgroup of $M$ has the same annihilator as $M$ if and only if $M$ is not torsion or $T(M)$ is elementary abelian.

\section{Applications to module algebras over Hopf alge- bras.}

Since we will apply the above module theoretic notions in this section to Hopf module algebras, we assume that the reader is familiar with the basic theory of Hopf algebras.

Let $R$ be a commutative ring and $H$ a Hopf algebra over $R$. Then $H$ is an $R$-algebra that admits a comultiplication $\Delta: H \rightarrow H \otimes H$ and a counit $\epsilon: H \rightarrow R$ which are $R$-algebra maps. We make free use of Sweedler's symbolic Sigma-notation for the comultiplication, i.e. for any $h \in H$ we write $\Delta(h)=\sum_{(h)} h_{1} \otimes h_{2}$. Moreover $S$ denotes the antipode of $H$, i.e. an anti-algebra map $S: H \rightarrow H$ such that $\epsilon(h) 1_{H}=\sum_{(h)} S\left(h_{1}\right) h_{2}=\sum_{(h)} h_{1} S\left(h_{2}\right)$ holds for all $h \in H$.

An $R$-algebra $A$ is called a left $H$-module algebra if $A$ is a left $H$-module such that $h \cdot(a b)=\sum_{(h)}\left(h_{1} \cdot a\right)\left(h_{2} \cdot b\right)$ and $h \cdot 1_{A}=\epsilon(h) 1_{A}$ for all $h \in H$ and $a, b \in A$, where ' '' denotes the action of $H$ on $A$. For any left $H$-module $M$ set $M^{H}:=\{m \in$ $M \mid h \cdot m=\epsilon(h) m \forall h \in H\}$. In particular $A^{H}$ becomes a subring of $A$, called the fixed ring of $A$. The smash product of $A$ and $H$ is the $R$-module $A \# H:=A \otimes H$ whose elements are finite sums of tensors $a \otimes h=: a \# h$. $A \# H$ becomes an $R$-algebra with the product: $(a \# h)(b \# g):=\sum_{(h)} a\left(h_{1} \cdot b\right) \# h_{2} . A$ is a subring of $A \# H$ and also a cyclic left $A \# H$-module, where an element $a \# h$ acts on an element $x \in A$ by $(a \# h) \cdot x:=a(h \cdot x)$. The left $A \# H$-submodules of $A$ are precisely the $H$-stable left ideals of $A$.

Let $M$ be a left $A \# H$-module. The map $\varphi_{M}: \operatorname{Hom}_{A \# H}(A, M) \rightarrow M^{H}$ with $\varphi_{M}(f):=\left(1_{A}\right) f$ for any $f \in \operatorname{Hom}_{A \# H}(A, M)$ is an isomorphism of $A^{H}$-modules. 
The collection of isomorphisms $\varphi_{M}$ are natural transformations between the functors $\operatorname{Hom}_{A \# H}(A,-)$ and $(-)^{H}$. Note that $\operatorname{End}_{A \# H}(A) \simeq A^{H}$ and $\operatorname{Hom}_{A \# H}(A, I) \simeq$ $I^{H}=I \cap A^{H}$ for any $H$-stable left ideal of $A$. We say that $A$ has a large fixed ring if $A^{H}$ intersects any non-zero $H$-stable left ideal non-trivially.

The module theoretic notions developed in the last sections have their equivalents in the case of Hopf actions as follows:

Lemma 7.1 let $A$ be an $H$-module algebra and $H$ a Hopf algebra over some commutative base ring $R$. Then

1. A is a retractable left $A \# H$-module if and only if $A$ has a large fixed ring.

2. $A$ is a semi-projective left $A \# H$-module if and only if $(A x)^{H}=A^{H} x$ for all $x \in A^{H}$.

3. $A$ is a self-projective left $A \# H$-module if and only if $(A / I)^{H}=A^{H} / I^{H}$ for all $H$-stable left ideals $I$ of $A$.

Proof. (1) is clear and (2) follows from the fact that $A$ is semi-projective as an $A \# H$-module if and only if for any $f \in \operatorname{End}_{A \# H}(A): \operatorname{Hom}_{A \# H}(A,(A) f)=$ $\operatorname{End}_{A \# H}(A) f$. Applying the correspondence between the functors $\operatorname{Hom}_{A \# H}(A,-)$ and $(-)^{H}$ we get $(A x)^{H}=A^{H} x$ for all $x \in A^{H}$.

(3) $A$ is self-projective if and only if $\operatorname{Hom}_{A \# H}(A,-)$ is exact on every short exact sequence

$$
0 \rightarrow I \rightarrow A \rightarrow A / I \rightarrow 0
$$

for any $H$-stable left ideal $I$ of $A$. Since the functor $\operatorname{Hom}_{A \# H}(A,-)$ is isomorphic to $(-)^{H}$ we get that $A$ is self-projective as an $A \# H$-module if and only if $(A / I)^{H} \simeq$ $A^{H} / I^{H}$ for all $H$-stable left ideals $I$ of $A$.

(4) See $[15]$.

A left integral of an Hopf algebra is an element $t \in H$ such that $h t=\epsilon(h) t$ for all $h \in H$. Left integrals are related to the finiteness of the Hopf algebra (see [14]). A module algebra $A$ is said to have an element of trace 1 if there exists an element $a \in A$ and a left integral $t$ such that $t \cdot a=1$. If $A$ contains an element of trace 1 then $A$ is a projective left $A \# H$-module. This happens in particular if $H$ is separable over $R$ (see $[14,4.8,5.8]$ ). If $H$ is a finite dimensional Hopf algebra over some field $R$ then $A$ is a projective left $A \# H$-module if and only if $A$ has an element of trace 1 (see $[14,4.8]$ ).

Since $I \cap A^{H}=I^{H}=\left(1_{A}\right) \operatorname{Hom}_{A \# H}(A, I)$ for any $H$-stable left ideal $I$ of $A$, we see that the $\star$-product $I \star J$ of two $H$-stable left ideals $I$ and $J$ of $A$ is equal to $I J^{H}$. We can now apply the characterisation of weakly compressible modules to the case of Hopf actions:

Proposition 7.2 Let $A$ be an $H$-module algebra and $H$ a Hopf algebra over some commutative base ring $R$. The following statements are equivalent:

(a) $A$ is a weakly compressible A\#H-module.

(b) A has a large fixed ring and $x(A x)^{H} \neq 0$ for all $0 \neq x \in A^{H}$.

(c) $\operatorname{Ann}_{I}\left(I^{H}\right)=0$ for all non-zero $H$-stable left ideals $I$ of $A$.

In this case $A$ is $H$-semiprime, i.e. $A$ does not contain any non-zero nilpotent $H$ stable ideal. 
Proof. $(a) \Leftrightarrow(b)$ follows from 5.1 and the correspondence between $(A x)^{H}$ and $\operatorname{Hom}_{A \# H}(A, A x)$.

Note that $\operatorname{Ann}_{I}\left(I^{H}\right)=I \cap \operatorname{Ann}_{A}\left(I^{H}\right)$. Since $I^{H}=(1) \operatorname{Hom}_{A \# H}(A, I)$, we have $a I^{H}=0$ if and only if $a \in \operatorname{Rej}(A, I)$ for all $a \in A$. Hence $\operatorname{Ann}_{A}\left(I^{H}\right)=\operatorname{Rej}(A, I)$ and $(a) \Leftrightarrow(c)$ also follows from 5.1.

Note that condition $(c)$ readily implies that if $A$ is weakly compressible, then it must be $H$-semiprime.

If $A$ is semi-projective as an $A \# H$-module the condition on $A$ of being weakly compressible is best described by $A$ having a large semiprime fixed ring.

Proposition 7.3 Suppose that $(A x)^{H}=A^{H} x$ holds for all $x \in A^{H}$. Then $A$ is a weakly compressible left $A \# H$-module if and only if $A$ has a large semiprime fixed ring.

Proof. This follows from 5.2.

Note that if $N$ is a fully invariant submodule of a module $M$ then $M / N$ is semi-projective (resp. self-projective) provided $M$ is semi-projective (resp. selfprojective). Moreover, if we let $\star^{\prime}$ and $\star$ denote the products in $\mathcal{L}(M / N)$ and $\mathcal{L}(M)$ respectively and assume that $M$ is self-projective, then for any submodules $K, L, N$ of $M$ with $N \subseteq K, L$ we have $K / N \star^{\prime} L / N=[(K \star L)+N] / N$.

Using the characterization of self-projective weakly compressible modules we can state the following:

Proposition 7.4 Suppose $(A / I)^{H}=\left(A^{H}+I\right) / I$ holds for all $H$-stable left ideals I. Then $A$ is a weakly compressible left $A \# H$-module if and only if $A$ is a subdirect product of $H$-module algebras with large prime fixed rings.

Proof. Let $L:=\{I \subseteq A \mid I$ is an $H$-stable ideal of $A\}$. Then $(L, \star)$ is an $\ell$ subgroupoid of $\left(\mathcal{L}\left({ }_{A \# H} A\right), \star\right)$. If $A$ is weakly compressible, then $(L, \star)$ is semiprime by 2.6 and $A$ is a subdirect product of prime elements of $(L, \star)$. Let $\left\{P_{i}\right\}$ be $H$ stable ideals that are prime elements in $(L, \star)$. The quotients $A / P_{i}$ are $H$-module algebras that are $\star$-prime self-projective left $A \# H$-modules. Since the $A \# H$-module structure of $A / P_{i}$ coincides with its $\left(A / P_{i}\right) \# H$-module structure each factor $A / P_{i}$ has a large prime fixed ring by 7.3. The converse follows from 5.7.

Finally we come to the case where $A$ is projective as an $A \# H$-module:

Theorem 7.5 Suppose $A$ is projective as a left $A \# H$-module. Then $A$ is a weakly compressible left $A \# H$-module if and only if $\operatorname{Ann}_{A \# H}(A)$ is a semiprime ideal.

As mentioned before $A$ is projective as $A \# H$-module if $H$ is separable over $R$ or if $H$ is finite dimensional over some field $R$ and $A$ contains an element of trace 1 .

If $A \# H$ is semiprime and $A$ is projective as an $A \# H$-module, we know by Amitsur's Proposition that $A$ has a large semiprime fixed ring (and hence $A$ is weakly compressible). If $A$ is weakly compressible then it is $H$-semiprime. It is an open question whether $A \# H$ is always semiprime for a semisimple Hopf algebra $H$ and an $H$-semiprime module algebra $A$.

Cohen and Fishman asked in [5] whether the smash product is semiprime provided $A$ is semiprime and $H$ is semisimple. For group actions this had been shown by Montgomery and Fisher [8]. It has also been shown in [15] that $A \# H$ is semiprime provided $A$ is a commutative semiprime module algebra over a semisimple Hopf algebra $H$ over a field of characteristic 0 . Those Hopf algebras $H$ such that $A \# H$ is semiprime provided $A$ is $H$-semiprime are called strongly semisimple. 
Corollary 7.6 Let $H$ be a strongly semisimple Hopf algebra over a ring $R$ and let $A$ be a left $H$-module algebra. Then the following statements are equivalent:

(a) A\#H is semiprime;

(b) A has a semiprime large fixed ring;

(c) A is weakly compressible A\#H-module;

(d) $A$ is $H$-semiprime.

\section{Acknowledgement}

I would like to express my gratitude to John Clark for having read an earlier version of this paper and for his many suggestions.

\section{References}

[1] R Ameri. On the prime submodules of multiplication modules. Int. J. Math. Math. Sci., (27):1715-1724, 2003.

[2] S.A. Amitsur. Rings of quotients and morita contexts. J. Algebra, 12:273-298, 1971.

[3] L. Bican, P. Jambor, T. Kepka, and P. Němec. Prime and coprime modules. Fund. Math., 57:33-45, 1980.

[4] G.D. Birkhoff. Lattice Theory. AMS, 1979.

[5] M. Cohen and D. Fishman. Hopf algebra actions. J. Algebra, 100:363-379, 1986.

[6] J. Dauns. Prime modules and one-sided ideals. Lecture Notes Pure Appl. Math., 55:301-344, 1980.

[7] J. Dauns. Semiprime modules and rings. Lecture Notes Math., 1448:41-62, 1990.

[8] J.W. Fisher and S. Montgomery. Semiprime skew group rings. J. Algebra, 52:241-247, 1978 .

[9] J. Jenkins and P.F. Smith. On the prime radical of a module over a commutative ring. Commun. Algebra, 20:3593-3602, 1992.

[10] J. Jirásko. Notes on generalized prime and coprime modules I. Comm. Math. Univ. Carolinae, 22(3):467-482, 1981.

[11] I. Kaplanski. Commutative rings. Allyn and Bacon, Boston, 1970.

[12] G. Khadzhiev and F. Callialp. On the differential prime radical of a differential ring. Turkish J. Math., 22:355-368, 1998.

[13] G. Khadzhiev and T.M. Shamilev. Complete $\ell$-groupoids and their prime spectra. Algebra and Logic, 36(3):204-211, 1997.

[14] C Lomp. Integrals in Hopf algebras over rings. to appear in Comm. Alg.

[15] C Lomp. When is a smash product semiprime? A partial answer. to appear in J.Algebra. 
[16] C. Lomp and A.J. Peña P. A note on prime modules. Divulg. Mat., 8(1):31-42, 2000 .

[17] I.V. Samsonova. Weakly compressible abelian groups. Russ. Math. Surv., 48(1):187-188, 1993.

[18] B. Soon-Sook. Modules with prime endomorphism rings. J. Korean Math. Soc., 38(5):987-1030, 2001.

[19] R. Van den Berg and R. Wisbauer. Duprime and dusemiprime modules. erscheint in J. Pure Appl. Algebra, 2000.

[20] J. Zelmanowitz. A class of modules with semisimple behavior. In A. Facchini and C. Menini, editors, Abelian Groups and Modules., pages 491-400. Kluwer Acad. Publ., 1995. 